Theoretical and Mathematical Physics, Vol. 124, No. 1, pp. $859-871$, 2000. Translated from Teoreticheskaya i Matematicheskaya Fizika, Vol. 124, No. 1, pp. 3-17, July 2000 (in Russian). 1 ]

\title{
ON THE SPECTRUM OF THE PERIODIC DIRAC OPERATOR
}

\author{
L.I. DANILOV
}

ABStRaCt. The absolute continuity of the spectrum for the periodic Dirac operator

$$
\widehat{D}=\sum_{j=1}^{n}\left(-i \frac{\partial}{\partial x_{j}}-A_{j}\right) \widehat{\alpha}_{j}+\widehat{V}^{(0)}+\widehat{V}^{(1)}, \quad x \in \mathbb{R}^{n}, \quad n \geq 3,
$$

is proved given that either $A \in C\left(\mathbb{R}^{n} ; \mathbb{R}^{n}\right) \cap H_{\mathrm{loc}}^{q}\left(\mathbb{R}^{n} ; \mathbb{R}^{n}\right), 2 q>n-2$, or the Fourier series of the vector potential $A: \mathbb{R}^{n} \rightarrow \mathbb{R}^{n}$ is absolutely convergent. Here, $\widehat{V}^{(s)}=\left(\widehat{V}^{(s)}\right)^{*}$ are continuous matrix functions and $\widehat{V}^{(s)} \widehat{\alpha}_{j}=(-1)^{s} \widehat{\alpha}_{j} \widehat{V}^{(s)}$ for all anticommuting Hermitian matrices $\widehat{\alpha}_{j}, \widehat{\alpha}_{j}^{2}=\widehat{I}, s=0,1$.

In [1], the absolute continuity of the spectrum for the periodic Dirac operator

$$
\widehat{D}=\sum_{j=1}^{n}\left(-i \frac{\partial}{\partial x_{j}}-A_{j}\right) \widehat{\alpha}_{j}+V \widehat{I}+V_{0} \widehat{\alpha}_{n+1}
$$

in $\mathbb{R}^{n}, n \geq 2$, was proved, where $V, V_{0} \in L_{\text {loc }}^{q}\left(\mathbb{R}^{2} ; \mathbb{R}\right), A \in L_{\text {loc }}^{q}\left(\mathbb{R}^{2} ; \mathbb{R}^{2}\right)$, $q>2$, for $n=2$ and $V, V_{0} \in C\left(\mathbb{R}^{n} ; \mathbb{R}\right), A \in C^{2 n+3}\left(\mathbb{R}^{n} ; \mathbb{R}^{n}\right)$ for $n \geq 3$. Here, $\widehat{\alpha}_{n+1}$ is a Hermitian matrix anticommuting with the matrices $\widehat{\alpha}_{j}, j=$ $1, \ldots, n$, and $\widehat{\alpha}_{n+1}^{2}=\widehat{I}$. For $n=2$, the proof is based on the results in [2,3], where the two-dimensional periodic Schrödinger operator was considered. In [3], the absolute continuity of the spectrum for this operator was proved in the case of the scalar (electric) and the vector (magnetic) potentials $V$ and $A$ satisfying the conditions $V \in L_{\text {loc }}^{q}\left(\mathbb{R}^{2} ; \mathbb{R}\right)$ and $A \in L_{\text {loc }}^{2 q}\left(\mathbb{R}^{2} ; \mathbb{R}^{2}\right)$, $q>1$. For the periodic Dirac operator with $n=2$, the same result as in [1] was independently obtained in [4]. However, it was assumed in [4] that $V_{0} \equiv m=$ const. But the functions $V_{0} \in L_{\text {loc }}^{q}\left(\mathbb{R}^{2} ; \mathbb{R}\right), q>2$, can in fact be considered in this case as well without any significant changes. The proof in [4] used the method suggested in [5], where the absolute continuity of the spectrum was established for the two-dimensional Dirac operator with the periodic potential $V \in L_{\text {loc }}^{q}\left(\mathbb{R}^{2} ; \mathbb{R}\right), q>2$ (and $A \equiv 0$ ). Sobolev's results (see [6]) for the absolute continuity of the spectrum of the Schrödinger

\footnotetext{
${ }^{1}$ In this version a few misprints have been corrected.

2000 Mathematics Subject Classification. Primary 35P05.
} 
operator with the periodic vector potential $A \in C^{2 n+3}\left(\mathbb{R}^{n} ; \mathbb{R}^{n}\right)$ were used in [1] for the case $n \geq 3$. Sobolev later replaced the last condition with the weaker condition $A \in H_{\mathrm{loc}}^{q}\left(\mathbb{R}^{n} ; \mathbb{R}^{n}\right), 2 q>3 n-2, n \geq 3$ (see the survey in [7]), which permitted changing the smoothness conditions on the vector potential $A$ for the periodic Dirac operator $[1,7]$ in an adequate manner. The absolute continuity of the spectrum for the Dirac operator in $\mathbb{R}^{n}$, $n \geq 3$, with the periodic scalar potential $V$ (for $A \equiv 0$ ) was proved in [8-10] under various constraints on $V$.

1. Let $\mathcal{L}_{M}, M \in \mathbb{N}$, denote the linear space of complex $M \times M$ matrices, let $\mathcal{S}_{M}$ be the set of Hermitian matrices in $\mathcal{L}_{M}$, and let the matrices $\widehat{\alpha}_{j} \in$ $\mathcal{S}_{M}, j=1, \ldots, n$, satisfy the commutation relations $\widehat{\alpha}_{j} \widehat{\alpha}_{l}+\widehat{\alpha}_{l} \widehat{\alpha}_{j}=2 \delta_{j l} \widehat{I}$, where $\widehat{I} \in \mathcal{L}_{M}$ is the identity matrix and $\delta_{j l}$ is the Kronecker delta. We write

$$
\begin{gathered}
\mathcal{L}_{M}^{(s)}=\left\{\widehat{L} \in \mathcal{L}_{M}: \widehat{L} \widehat{\alpha}_{j}=(-1)^{s} \widehat{\alpha}_{j} \widehat{L} \text { for all } j=1, \ldots, n\right\}, \\
\mathcal{S}_{M}^{(s)}=\mathcal{L}_{M}^{(s)} \cap \mathcal{S}_{M}, \quad s=0,1 .
\end{gathered}
$$

We consider the Dirac operator

$$
\widehat{D}=\widehat{D}_{0}+\widehat{V}^{(0)}+\widehat{V}^{(1)}-\sum_{j=1}^{n} A_{j} \widehat{\alpha}_{j}=\sum_{j=1}^{n}\left(-i \frac{\partial}{\partial x_{j}}-A_{j}\right) \widehat{\alpha}_{j}+\widehat{V}^{(0)}+\widehat{V}^{(1)},
$$

where $n \geq 3\left(i^{2}=-1\right)$. The vector function $A: \mathbb{R}^{n} \rightarrow \mathbb{R}^{n}$ and the matrix functions $\widehat{V}^{(s)}: \mathbb{R}^{n} \rightarrow \mathcal{S}_{M}^{(s)}, s=0,1$, are assumed to be periodic with a period lattice $\Lambda \subset \mathbb{R}^{n}$. We set

$$
\widehat{V}=\widehat{V}^{(0)}+\widehat{V}^{(1)}-\sum_{j=1}^{n} A_{j} \widehat{\alpha}_{j}
$$

The coordinates of the vectors in $\mathbb{R}^{n}$ are set in an orthogonal basis $\left\{\mathcal{E}_{j}\right\}$. Here, $E_{j}$ and $E_{j}^{*}$ are the basis vectors in the lattice $\Lambda$ and its reciprocal lattice $\Lambda^{*},\left(E_{j}, E_{l}^{*}\right)=\delta_{j l}(|$.$| and (.,$.$) are the length and the inner product$ of vectors in $\mathbb{R}^{n}$ ),

$$
\begin{aligned}
K & =\left\{x=\sum_{j=1}^{n} \xi_{j} E_{j}: 0 \leq \xi_{j}<1, j=1, \ldots, n\right\}, \\
K^{*} & =\left\{y=\sum_{j=1}^{n} \eta_{j} E_{j}: 0 \leq \eta_{j}<1, j=1, \ldots, n\right\},
\end{aligned}
$$

and $v(K)$ and $v\left(K^{*}\right)$ are the volumes of the elementary cells $K$ and $K^{*}$.

The inner products and the norms in the spaces $L^{2}\left(K ; \mathbb{C}^{M}\right)$ and $\mathbb{C}^{M}$ are introduced in the usual way with (as a rule) the usual notation (without 
indicating the spaces themselves). The matrices in $\mathcal{L}_{M}$ are identified with the operators on the space $\mathbb{C}^{M}$ (and their norm is defined as the norm of operators on $\left.\mathbb{C}^{M}\right)$. Let $H^{q}\left(\mathbb{R}^{n} ; \mathbb{C}^{d}\right), d \in \mathbb{N}$, be the Sobolev class of order $q \geq 0$, and let $\widetilde{H}^{q}\left(K ; \mathbb{C}^{d}\right)$ be the set of vector functions $\phi: K \rightarrow \mathbb{C}^{d}$ whose periodic extensions (with the period lattice $\Lambda$ ) belong to $H_{\text {loc }}^{q}\left(\mathbb{R}^{n} ; \mathbb{C}^{d}\right)$. In what follows, the functions defined on the elementary cell $K$ are identified with their periodic extensions throughout the space $\mathbb{R}^{n}$.

We let

$$
\chi_{N}=v^{-1}(K) \int_{K} \chi(x) e^{-2 \pi i(N, x)} d^{n} x, \quad N \in \Lambda^{*},
$$

denote the Fourier coefficients of the functions $\chi \in L^{1}(K, U)$, where $U$ is the space $\mathbb{C}$ or $\mathbb{C}^{M}$ or $\mathcal{L}_{M}$.

Let $\mathcal{B}(\mathbb{R})$ be the set of Borel subsets $\mathcal{O} \subseteq \mathbb{R}$, and let $\mathcal{M}_{h}, h>0$, be the set of signed even Borel measures (charges) $\mu: \mathcal{B}(\mathbb{R}) \rightarrow \mathbb{R}$ such that

$$
\begin{gathered}
\widehat{\mu}(p)=\int_{\mathbb{R}} e^{i p t} d \mu(t)=1 \text { for }|p| \leq 2 \pi h, \quad p \in \mathbb{R}, \\
\|\mu\|=\sup _{\mathcal{O} \in \mathcal{B}(\mathbb{R})}(|\mu(\mathcal{O})|+|\mu(\mathbb{R} \backslash \mathcal{O})|)<+\infty, \quad \mu \in \mathcal{M}_{h} .
\end{gathered}
$$

For an arbitrary vector $\gamma \in \Lambda \backslash\{0\}$, an arbitrary measure $\mu \in \mathcal{M}_{h}, h>0$, and any vector $\widetilde{e} \in S_{n-2}\left(|\gamma|^{-1} \gamma\right)=\left\{e^{\prime} \in S_{n-1}:\left(\gamma, e^{\prime}\right)=0\right\}$, where $S_{n-1}$ is the unit sphere in $\mathbb{R}^{n}$, we write

$$
\widetilde{A}(\gamma, \mu, \widetilde{e} ; x)=\int_{\mathbb{R}} d \mu(t) \int_{0}^{1} A(x-\xi \gamma-t \widetilde{e}) d \xi, \quad x \in \mathbb{R}^{n} .
$$

In this paper, we consider continuous (periodic) functions $A: \mathbb{R}^{n} \rightarrow$ $\mathbb{R}^{n}$ and $\widehat{V}^{(s)}: \mathbb{R}^{n} \rightarrow \mathcal{S}_{M}^{(s)}, s=0,1$. In this case, $\widehat{D}=\widehat{D}_{0}+\widehat{V}$ is a self-adjoint operator on the Hilbert space $L^{2}\left(\mathbb{R}^{n} ; \mathbb{C}^{M}\right)$ with the domain $D(\widehat{D})=D\left(\widehat{D}_{0}\right)=H^{1}\left(\mathbb{R}^{n} ; \mathbb{C}^{M}\right)$.

Theorem 1. Let $A: \mathbb{R}^{n} \rightarrow \mathbb{R}^{n}$ and $\widehat{V}^{(s)}: \mathbb{R}^{n} \rightarrow \mathcal{S}_{M}^{(s)}, s=0,1$, be continuous periodic functions with the period lattice $\Lambda \subset \mathbb{R}^{n}, n \geq 3$. If

$$
\max _{\tilde{e} \in S_{n-2}\left(|\gamma|^{-1} \gamma\right)}\left\|\left|\widetilde{A}(\gamma, \mu, \widetilde{e} ; .)-A_{0}\right|\right\|_{L^{\infty}\left(\mathbb{R}^{n}\right)}<\pi|\gamma|^{-1}
$$

for some vector $\gamma \in \Lambda \backslash\{0\}$ and a measure $\mu \in \mathcal{M}_{h}, h>0$, where

$$
A_{0}=v^{-1}(K) \int_{K} A(x) d^{n} x,
$$


then the spectrum of operator (1) is absolutely continuous.

The operator $\widehat{D}$ is unitarily equivalent to the direct integral

$$
\int_{2 \pi K^{*}}^{\oplus} \widehat{D}(k) \frac{d^{n} k}{(2 \pi)^{n} v\left(K^{*}\right)},
$$

where

$$
\begin{gathered}
\widehat{D}(k)=\widehat{D}_{0}(k)+\widehat{V}, \quad \widehat{D}_{0}(k)=\sum_{j=1}^{n}\left(-i \frac{\partial}{\partial x_{j}}+k_{j}\right) \widehat{\alpha}_{j}, \quad k_{j}=\left(k, \mathcal{E}_{j}\right), \\
D(\widehat{D}(k))=D\left(\widehat{D}_{0}(k)\right)=\widetilde{H}^{1}\left(K ; \mathbb{C}^{M}\right) \subset L^{2}\left(K ; \mathbb{C}^{M}\right) .
\end{gathered}
$$

The vector $k \in \mathbb{R}^{n}$ is called a quasimomentum. The unitary equivalence is established using the Gel'fand transformation [11] (also see [9] for the case of the periodic Dirac operator). The self-adjoint operators $\widehat{D}(k)$ have compact resolvents and hence discrete spectra. Let $E_{\nu}(k), \nu \in \mathbb{Z}$, be the eigenvalues of the operators $\widehat{D}(k)$. We assume that they are arranged in an increasing order (counting multiplicities). The eigenvalues can be indexed for different $k$ such that the functions $\mathbb{R}^{n} \ni k \rightarrow E_{\nu}(k)$ are continuous.

Let $e \in S_{n-1}$. For $k \in \mathbb{R}^{n}$ and $\varkappa \geq 0$, we write

$$
\begin{gathered}
\widehat{D}_{0}(k+i \varkappa e)=\widehat{D}_{0}(k)+i \varkappa \sum_{j=1}^{n} e_{j} \widehat{\alpha}_{j}, \quad e_{j}=\left(e, \mathcal{E}_{j}\right), \\
\widehat{D}(k+i \varkappa e)=\widehat{D}_{0}(k+i \varkappa e)+\widehat{V}, \\
D(\widehat{D}(k+i \varkappa e))=D\left(\widehat{D}_{0}(k+i \varkappa e)\right)=\widetilde{H}^{1}\left(K ; \mathbb{C}^{M}\right) .
\end{gathered}
$$

Proof of Theorem 1. We use the Thomas method [12]. Because it is well known $[4,9]$ (see $[2,13]$ for the case of the periodic Schrödinger operator), we present only a brief scheme of the method. The decomposition of the operator $\widehat{D}$ into direct integral (3) and the piecewise analyticity of the functions $\mathbb{R} \ni \xi \rightarrow E_{\nu}(k+\xi e), \nu \in \mathbb{Z}, k \in \mathbb{R}^{n}$, imply (see Theorems XIII.85 and XIII.86 in [13]) that to prove the absolute continuity of the spectrum of operator (1), it suffices to show that the functions $\xi \rightarrow E_{\nu}(k+\xi e)$ are not constant (for some unit vector $e$ ) on every interval $\left(\xi_{1}, \xi_{2}\right) \subset \mathbb{R}$. But if we suppose that $E_{\nu}(k+\xi e) \equiv E$ for all $\xi \in\left(\xi_{1}, \xi_{2}\right), \xi_{1}<\xi_{2}$, then it follows from the analytic Fredholm theorem that $E$ is an eigevalue of $\widehat{D}(k+(\xi+i \varkappa) e)$ for all $\xi+i \varkappa \in \mathbb{C}$. Consequently, it suffices to prove the invertibility of the operators $\widehat{D}(k+(\xi+i \varkappa) e)-E, k \in \mathbb{R}^{n}, E \in \mathbb{R}$, for some $\xi+i \varkappa \in \mathbb{C}$. Theorem 1 is therefore a consequence of the following assertion. 
Theorem 2. Let $\gamma \in \Lambda \backslash\{0\}, e=|\gamma|^{-1} \gamma, \mu \in \mathcal{M}_{h}, h>0$. Let $A: \mathbb{R}^{n} \rightarrow$ $\mathbb{C}^{n}$ and $\widehat{V}^{(s)}: \mathbb{R}^{n} \rightarrow \mathcal{L}_{M}^{(s)}, s=0,1$, be continuous periodic functions with the period lattice $\Lambda \subset \mathbb{R}^{n}, n \geq 3$. If $A_{0}=0$ and

$$
\max _{\widetilde{e} \in S_{n-2}\left(|\gamma|^{-1} \gamma\right)}\|(\widetilde{A}(\gamma, \mu, \widetilde{e} ; .), \widetilde{e})+i(\widetilde{A}(\gamma, \mu, \widetilde{e} ; .), e)\|_{L^{\infty}\left(\mathbb{R}^{n}\right)}=\widetilde{\theta} \pi|\gamma|^{-1},
$$

where $\tilde{\theta} \in[0,1)$, then for any $\theta \in(0,1-\widetilde{\theta})$, there exists a number $\varkappa_{0}=$ $\varkappa_{0}(\gamma, h, \mu ; \widehat{V}, \theta)>0$ such that the inequality

$$
\|\widehat{D}(k+i \varkappa e) \phi\| \geq \theta \pi|\gamma|^{-1} \exp \left(-4 C\|\mu\| \max \left\{|\gamma|, h^{-1}\right\}\|A\|_{L^{\infty}\left(\mathbb{R}^{n} ; \mathbb{C}^{n}\right)}\right)\|\phi\|
$$

holds for all $k \in \mathbb{R}^{n}$ with $(k, \gamma)=\pi$, all $\varkappa \geq \varkappa_{0}$, and all vector functions $\phi \in \widetilde{H}^{1}\left(K ; \mathbb{C}^{M}\right)$, where $C>0$ is a universal constant to be defined in Lemma 1.

Theorem 2 is proved in Section 3. The following theorem is a consequence of Theorem 1 .

Theorem 3. Let $A: \mathbb{R}^{n} \rightarrow \mathbb{R}^{n}$ and $\widehat{V}^{(s)}: \mathbb{R}^{n} \rightarrow \mathcal{S}_{M}^{(s)}, s=0,1$, be continuous periodic functions with the period lattice $\Lambda \subset \mathbb{R}^{n}, n \geq 3$. If at least one of the conditions

1. $A \in H_{\mathrm{loc}}^{q}\left(\mathbb{R}^{n} ; \mathbb{R}^{n}\right), 2 q>n-2$, or

2. $\sum_{N \in \Lambda^{*}}\left\|A_{N}\right\|_{\mathbb{C}^{n}}<+\infty$

holds, then the spectrum of operator (1) is absolutely continuous.

Theorem 4 is used to prove Theorem 3.

Theorem 4. Let $\Lambda$ be a lattice in $\mathbb{R}^{n}, n \geq 2$. There are positive constants $c_{1}$ and $c_{2}$ depending on $n$ and $\Lambda$ such that for any nonnegative Borel measure $\mu$ on the unit sphere $S_{n-1} \subset \mathbb{R}^{n}$, any $h>0$, and any $R_{0} \geq \min _{\gamma \in \Lambda \backslash\{0\}}|\gamma|$, there exists a vector $\gamma \in \Lambda \backslash\{0\}$ such that

1. $|\gamma| \leq R_{0}$,

2. if $\left(\gamma, \gamma^{\prime}\right)=0$ for some vector $\gamma^{\prime} \in \Lambda \backslash\{0\}$, then $\left|\gamma^{\prime}\right|>c_{1} R_{0}^{1 /(n-1)}\left(\Lambda^{*}\right.$ is the reciprocal lattice of $\Lambda)$,

3. $\mu\left(\left\{e^{\prime} \in S_{n-1}:\left|\left(e^{\prime}, \gamma\right)\right| \leq h\right\}\right) \leq c_{2}|\gamma|^{-1} \max \left\{h, R_{0}^{-1 /(n-1)}\right\} \mu\left(S_{n-1}\right)$.

The proof of Theorem 4 for the lattice $\Lambda=\mathbb{Z}^{n}$ and for $h=c_{3} R_{0}^{-1 /(n-1)}$ (where $c_{3}=c_{3}(n)>0$ ) is presented in [14] (see [15] for $n=3$ ). The proof in the general case follows the one suggested in [14] with some slight changes.

Proof of Theorem 3. It can be assumed that $A_{0}=0$. We write $F(A ; \gamma, \mu)=\max _{\widetilde{e} \in S_{n-2}\left(|\gamma|^{-1} \gamma\right)}|\gamma|\|\widetilde{A}(\gamma, \mu, \widetilde{e} ; .)\|_{L^{\infty}\left(\mathbb{R}^{n} ; \mathbb{R}^{n}\right)}, \quad \gamma \in \Lambda \backslash\{0\}, \quad \mu \in \mathcal{M}_{h}$. 
Let condition 1 hold. We define the measure

$$
\mu^{(1)}(.)=\sum_{N \in \Lambda^{*} \backslash\{0\}}|N|^{2 q}\|A\|_{\mathbb{C}^{n} \delta_{N /|N|}^{2}(.)}
$$

on the unit sphere $S_{n-1}$, where $\delta_{e^{\prime}}($.$) is the Dirac measure concentrated$ at the point $e^{\prime} \in S_{n-1}$. From Theorem 4 (applied to the measure $\mu^{(1)}$ ), it follows that for any $R_{0} \geq \min _{\gamma \in \Lambda \backslash\{0\}}|\gamma|$ there is a vector $\gamma \in \Lambda \backslash\{0\}$ such that $|\gamma| \leq R_{0}$

$$
\sum_{N \in \Pi(\gamma)}|N|^{2 q}\left\|A_{N}\right\|_{\mathbb{C}^{n}}^{2} \leq c_{2}|\gamma|^{-1} R_{0}^{-1 /(n-1)} \sum_{N \in \Lambda^{*}}|N|^{2 q}\left\|A_{N}\right\|_{\mathbb{C}^{n}}^{2}
$$

and $\left|\gamma^{\prime}\right|>c_{1} R_{0}^{1 /(n-1)}$ for all $\gamma^{\prime} \in \Pi(\gamma) \doteq\left\{\gamma^{\prime} \in \Lambda^{*} \backslash\{0\}:\left(\gamma, \gamma^{\prime}\right)=0\right\}$. We take a measure $\mu \in \mathcal{M}_{h}$ (for some $h>0$ ) such that $|\widehat{\mu}(p)| \leq 1$ for all $p \in \mathbb{R}$ and $\widehat{\mu}(p)=0$ if $|p| \geq 2 \pi h_{1}>2 \pi h$. For a vector $\widetilde{e} \in S_{n-2}\left(|\gamma|^{-1} \gamma\right)$, we write $\Pi(\gamma, \widetilde{e})=\left\{\gamma^{\prime} \in \Pi(\gamma):\left|\left(\gamma^{\prime}, \widetilde{e}\right)\right| \leq h_{1}\right\}$. Because $2 q>n-2$, we have

$$
\sum_{N \in \Pi(\gamma, \widetilde{e})}|N|^{-2 q} \leq c_{4} R_{0}^{-2 q /(n-1)}
$$

for all $\widetilde{e} \in S_{n-2}\left(|\gamma|^{-1} \gamma\right)$, where the constant $c_{4}>0$ depends on $n, \Lambda, q$, and $h_{1}$. Consequently,

$$
\begin{gathered}
F(A ; \gamma, \mu) \leq \sup _{\widetilde{e} \in S_{n-2}\left(|\gamma|^{-1} \gamma\right)}|\gamma| \sum_{N \in \Pi(\gamma, \widetilde{e})}\left\|A_{N}\right\|_{\mathbb{C}^{n}} \leq \\
|\gamma|\left(\sup _{\tilde{e} \in S_{n-2}\left(|\gamma|^{-1} \gamma\right)} \sum_{N \in \Pi(\gamma, \widetilde{e})}|N|^{-2 q}\right)^{1 / 2}\left(\sum_{N \in \Pi(\gamma)}|N|^{2 q}\left\|A_{N}\right\|_{\mathbb{C}^{n}}^{2}\right)^{1 / 2} \leq \\
\sqrt{c_{2} c_{4}} R_{0}^{(n-2-2 q) /(2(n-1))}\left(\sum_{N \in \Lambda^{*}}|N|^{2 q}\left\|A_{N}\right\|_{\mathbb{C}^{n}}^{2}\right)^{1 / 2} \cdot
\end{gathered}
$$

The right-hand side of (4) becomes arbitrarily small if a sufficiently large number $R_{0}$ is chosen (and inequality (2) consequently holds). Case 2, for which the Dirac measure $\mu=\delta$ is chosen, is considered in a similar (slightly simpler) way. Theorem 3 is proved.

2. We fix a vector $\gamma \in \Lambda \backslash\{0\}$ and a measure $\mu \in \mathcal{M}_{h}, h>0, e=|\gamma|^{-1} \gamma$. In what follows, the constants we introduce can depend on $\gamma, h$, and $\mu$, but we do not indicate this dependence explicitly (untill Theorem 8 below).

Let $\widehat{P}^{\mathcal{C}}$, where $\mathcal{C} \subseteq \Lambda^{*}$, denote the orthogonal projection on $L^{2}\left(K ; \mathbb{C}^{M}\right)$ that takes a vector function $\phi \in L^{2}\left(K ; \mathbb{C}^{M}\right)$ to the vector function

$$
\widehat{P}^{\mathcal{C}} \phi=\phi^{\mathcal{C}}=\sum_{N \in \mathcal{C}} \phi_{N} e^{2 \pi i(N, x)}
$$


(here, $\left.\phi^{\emptyset} \equiv 0\right)$. We introduce the notation $\mathcal{H}(\mathcal{C})=\left\{\phi \in L^{2}\left(K ; \mathbb{C}^{M}\right)\right.$ : $\phi_{N}=0$ for $\left.N \notin \mathcal{C}\right\}$.

Let $\mathcal{P}(e)=\{\tau e: \tau \in \mathbb{R}\}$. For the vectors $x \in \mathbb{R}^{n} \backslash \mathcal{P}(e)$, we write

$$
\widetilde{e}(x)=(x-(x, e) e)|x-(x, e) e|^{-1} \in S_{n-2}(e),
$$

where $S_{n-2}(e)=\left\{\widetilde{e} \in S_{n-1}:(e, \widetilde{e})=0\right\}$; we also write $\sigma_{n-2}=\operatorname{mes}\left(S_{n-2}\right)$, where mes (.) is the standard measure ('surface area') on the unit sphere $S_{n-2}=S_{n-2}(e)$. For $\beta>0$ and $\varkappa>\beta$, we write

$$
\begin{gathered}
\mathcal{O}_{\beta}=\mathcal{O}_{\beta}(\varkappa)=\left\{x \in \mathbb{R}^{n}:|(x, e)|<\beta \text { and }|\varkappa-| x-(x, e) e||<\beta\right\}, \\
\mathcal{K}_{\beta}=\mathcal{K}_{\beta}(k ; \varkappa)=\left\{N \in \Lambda^{*}: k+2 \pi N \in \mathcal{O}_{\beta}\right\}, \quad k \in \mathbb{R}^{n} .
\end{gathered}
$$

We set

$$
\widehat{P}_{\widetilde{e}}^{ \pm}=\frac{1}{2}\left(\widehat{I} \mp i\left(\sum_{j=1}^{n} e_{j} \widehat{\alpha}_{j}\right)\left(\sum_{j=1}^{n} \widetilde{e}_{j} \widehat{\alpha}_{j}\right)\right)
$$

for all $\widetilde{e} \in S_{n-2}(e)$, where $\widehat{P}_{\widetilde{e}}^{ \pm}$are orthogonal projections on $\mathbb{C}^{M}$.

For $k \in \mathbb{R}^{n}, \varkappa \geq 0$, and $N \in \Lambda^{*}$, we introduce the notation

$$
\begin{gathered}
\widehat{D}_{N}(k ; \varkappa)=\sum_{j=1}^{n}\left(k_{j}+2 \pi N_{j}+i \varkappa e_{j}\right) \widehat{\alpha}_{j}, \\
G_{N}^{ \pm}(k ; \varkappa)=\left((k+2 \pi N, e)^{2}+\left(\varkappa \pm \sqrt{|k+2 \pi N|^{2}-(k+2 \pi N, e)^{2}}\right)^{2}\right)^{1 / 2},
\end{gathered}
$$

and $G_{N}(k ; \varkappa)=G_{N}^{-}(k ; \varkappa)$. The inequalities

$$
G_{N}(k ; \varkappa)\|u\| \leq\left\|\widehat{D}_{N}(k ; \varkappa) u\right\| \leq G_{N}^{+}(k ; \varkappa)\|u\|, \quad u \in \mathbb{C}^{M},
$$

hold. If $(k, \gamma)=\pi$, then $G_{N}(k ; \varkappa) \geq|(k+2 \pi N, e)| \geq \pi|\gamma|^{-1}$. For all vector functions $\phi \in \widetilde{H}^{1}\left(K ; \mathbb{C}^{M}\right)$,

$$
\widehat{D}_{0}(k+i \varkappa e) \phi=\sum_{N \in \Lambda^{*}} \widehat{D}_{N}(k ; \varkappa) \phi_{N} e^{2 \pi i(N, x)} .
$$

In this case (for all $\varkappa \geq 0$ and $k+2 \pi N \notin \mathcal{P}(e)$ ), we have

$$
\left\|\widehat{D}_{N}(k ; \varkappa) \widehat{P}_{\widetilde{e}(k+2 \pi N)}^{ \pm} \phi_{N}\right\|=G_{N}^{ \pm}(k ; \varkappa)\left\|\widehat{P}_{\widetilde{e}(k+2 \pi N)}^{ \pm} \phi_{N}\right\|,
$$

and

$$
\widehat{P}_{\widetilde{e}(k+2 \pi N)}^{ \pm} \widehat{D}_{N}(k ; \varkappa) \widehat{P}_{\widetilde{e}(k+2 \pi N)}^{ \pm}=\widehat{O},
$$

where $\widehat{O} \in \mathcal{L}_{M}$ is the zero matrix.

We let $\widehat{P}^{ \pm}=\widehat{P}^{ \pm}(k)$, where $k \in \mathbb{R}^{n}$, denote the operators on $L^{2}\left(K ; \mathbb{C}^{M}\right)$ that take vector functions $\phi \in L^{2}\left(K ; \mathbb{C}^{M}\right)$ to the vector functions $\widehat{P}^{ \pm} \phi \in$ 
$L^{2}\left(K ; \mathbb{C}^{M}\right)$ with the Fourier coefficients $\left(\widehat{P}^{ \pm} \phi\right)_{N}=\widehat{P}_{\widetilde{e}(k+2 \pi N)}^{ \pm} \phi_{N}$ if $k+$ $2 \pi N \notin \mathcal{P}(e)$ and $\left(\widehat{P}^{ \pm} \phi\right)_{N}=0$ otherwise.

For the matrix function $\widehat{V}=\widehat{V}^{(0)}+\widehat{V}^{(1)}-\sum_{j=1}^{n} A_{j} \widehat{\alpha}_{j}$, where $\widehat{V}^{(s)}: \mathbb{R}^{n} \rightarrow$ $\mathcal{L}_{M}^{(s)}, s=0,1$, and $A: \mathbb{R}^{n} \rightarrow \mathbb{C}^{n}$ are continuous periodic functions with the period lattice $\Lambda$, we write

$$
W=W(\widehat{V})=n\|A\|_{L^{\infty}\left(\mathbb{R}^{n} ; \mathbb{C}^{n}\right)}+\sum_{s=0,1}\left\|\widehat{V}^{(s)}\right\|_{L^{\infty}\left(\mathbb{R}^{n} ; \mathcal{L}_{M}\right)}
$$

We set $c_{5}(A)=c_{5}(A ; \gamma, h, \mu)=\exp \left(-4 C\|\mu\| \max \left\{|\gamma|, h^{-1}\right\}\|A\|_{L^{\infty}\left(\mathbb{R}^{n} ; \mathbb{C}^{n}\right)}\right)$, where $C>0$ is a universal constant to be defined in Lemma 1.

Theorem 5. Let $\widetilde{\theta} \in[0,1), \theta \in(0,1-\widetilde{\theta}), W_{0} \geq 0, R \geq 1, \beta>0$, and $a \in(0,1]$. Also, let us fix a vector $\gamma \in \Lambda \backslash\{0\}$, a number $h>0$, and $a$ measure $\mu \in \mathcal{M}_{h} ; e=|\gamma|^{-1} \gamma$. Then there are numbers $b=b\left(\widetilde{\theta}, \theta, W_{0} ; a\right)>$ 0 and $\varkappa_{0}=\varkappa_{0}\left(\widetilde{\theta}, \theta, W_{0}, R, \beta ; a\right)>4 \beta+R$ such that the inequality

$$
\begin{aligned}
& \left\|\widehat{P}^{+}(k) \widehat{D}(k+i \varkappa e) \phi\right\|^{2}+a^{2}\left\|\widehat{P}^{-}(k) \widehat{D}(k+i \varkappa e) \phi\right\|^{2} \geq \\
& c_{5}^{2}(A)\left(\left(\theta \frac{\pi}{|\gamma|}\right)^{2}\left\|P^{-}(k) \phi\right\|^{2}+\left(\frac{b \varkappa}{\beta+R}\right)^{2}\left\|P^{+}(k) \phi\right\|^{2}\right)
\end{aligned}
$$

holds for all vectors $k \in \mathbb{R}^{n}$ with $(k, \gamma)=\pi$, all $\varkappa \geq \varkappa_{0}$, all continuous periodic functions $\widehat{V}^{(s)}: \mathbb{R}^{n} \rightarrow \mathcal{L}_{M}^{(s)}, s=0,1$, and $A: \mathbb{R}^{n} \rightarrow \mathbb{C}^{n}$ (with the period lattice $\Lambda \subset \mathbb{R}^{n}, n \geq 3$ ) such that $A_{0}=0$,

$$
\begin{gathered}
W(\widehat{V}) \leq W_{0}, \\
\max _{\widetilde{e} \in S_{n-2}(e)}\|(\widetilde{A}(\gamma, \mu, \widetilde{e} ; .), \widetilde{e})+i(\widetilde{A}(\gamma, \mu, \widetilde{e} ; .), e)\|_{L^{\infty}\left(\mathbb{R}^{n}\right)} \leq \widetilde{\theta} \pi|\gamma|^{-1}, \\
\widehat{V}_{N}=0 \text { for } 2 \pi|N|>R,
\end{gathered}
$$

and all vector functions $\phi \in \mathcal{H}\left(\mathcal{K}_{\beta}(k ; \varkappa)\right)$.

Proof. Without loss of generality we assume that the basis vector $\mathcal{E}_{2}$ coincides with $e$. We fix some numbers $\theta<\theta_{4}<\theta_{3}<\theta_{2}<\theta_{1}<1-\widetilde{\theta}$ and write $\delta=1-\theta_{4}^{2} \theta_{3}^{-2}$ and $c_{5}^{\prime}=\exp \left(-4 C\|\mu\| \max \left\{|\gamma|, h^{-1}\right\} W_{0}\right)$. We choose a number $\widetilde{\varepsilon} \in(0,1)$ proceeding from the condition $\left(c_{5}^{\prime}\right)^{2}\left((1-\widetilde{\varepsilon}) \theta_{4}^{2}-\right.$ $\left.\theta^{2}\right) \pi^{2}|\gamma|^{-2} \geq 2 \delta^{-1} W_{0}^{2} \widetilde{\varepsilon}$. Lower bounds for the constant $\varkappa_{0}$ are specified in the course of the proof. We first suppose that $\varkappa_{0}>4 \beta+R$. In this case, if $N \in \mathcal{K}_{\beta}(k ; \varkappa), k \in \mathbb{R}^{n}, \varkappa \geq \varkappa_{0}$, and $2 \pi\left|N^{\prime}\right| \leq R$ (where $N^{\prime} \in \Lambda^{*}$ ), then $\left|\widetilde{e}\left(k+2 \pi\left(N+N^{\prime}\right)\right)-\widetilde{e}(k+2 \pi N)\right|<2 R / \varkappa$. There is a number $c_{6}=$ $c_{6}(\widetilde{\varepsilon})>0$ such that for all $\varkappa \geq \varkappa_{0}$, there are nonintersecting (nonempty) 
open sets $\widetilde{\Omega}_{\lambda}=\widetilde{\Omega}_{\lambda}(\varkappa) \subset S_{n-2}=S_{n-2}(e)$ and vectors $E^{\lambda}=E^{\lambda}(\varkappa) \in \widetilde{\Omega}_{\lambda}$, $\lambda=1, \ldots, \lambda(\widetilde{\varepsilon}, R ; \varkappa)$, such that

1. $\left|\widetilde{e}-E^{\lambda}\right| \leq \widetilde{\rho}=c_{6} R / \varkappa$ for all $\widetilde{e} \in \widetilde{\Omega}_{\lambda}$;

2. $\left|\widetilde{e}^{\prime}-\widetilde{e}^{\prime \prime}\right|>8 R / \varkappa$ for all $\widetilde{e}^{\prime} \in \widetilde{\Omega}_{\lambda_{1}}, \widetilde{e}^{\prime \prime} \in \widetilde{\Omega}_{\lambda_{2}}, \lambda_{1} \neq \lambda_{2}$;

3. $\operatorname{mes}\left(S_{n-2} \backslash \bigcup_{\lambda} \widetilde{\Omega}_{\lambda}\right)<(1 / 2) \widetilde{\varepsilon} \sigma_{n-2}$.

We introduce the notation $\rho=\widetilde{\rho}+2 R / \varkappa, \rho^{\prime}=\widetilde{\rho}+4 R / \varkappa$. Let

$$
\Omega_{\lambda}=\left\{\widetilde{e} \in S_{n-2}:\left|\widetilde{e}-\widetilde{e}^{\prime}\right|<\frac{2 R}{\varkappa} \text { for some } \widetilde{e}^{\prime} \in \widetilde{\Omega}_{\lambda}\right\}
$$

$\widetilde{\Omega}_{\lambda} \subset \Omega_{\lambda}$, and $\left|\widetilde{e}^{\prime}-\widetilde{e}^{\prime \prime}\right|>4 R / \varkappa$ for all $\widetilde{e}^{\prime} \in \Omega_{\lambda_{1}}, \widetilde{e}^{\prime \prime} \in \Omega_{\lambda_{2}}, \lambda_{1} \neq \lambda_{2}$. Property 3 implies that for any $k \in \mathbb{R}^{n}$ with $(k, \gamma)=\pi$, any $\varkappa \geq \varkappa_{0}$, and any $\phi \in \mathcal{H}\left(\mathcal{K}_{\beta}(k ; \varkappa)\right)$, there is an orthogonal transformation $\widehat{S}=\widehat{S}(k, \varkappa ; \phi)$ of the unit sphere $S_{n-2}$ such that (for each of the signs)

$$
\sum_{N \in \mathcal{K}_{\beta}: \widetilde{e}(k+2 \pi N) \notin \bigcup_{\lambda} \widehat{S} \widetilde{\Omega}_{\lambda}}\left\|\widehat{P}_{\widetilde{e}(k+2 \pi N)}^{ \pm} \phi_{N}\right\|^{2} \leq \widetilde{\varepsilon} v^{-1}(K)\left\|\widehat{P}^{ \pm} \phi\right\|^{2} .
$$

We write

$$
\begin{gathered}
\widetilde{e}^{\lambda}=\widehat{S}(k, \varkappa ; \phi) E^{\lambda}, \\
\widetilde{\mathcal{K}}_{\beta}^{\lambda}=\widetilde{\mathcal{K}}_{\beta}^{\lambda}(k, \varkappa ; \phi)=\left\{N \in \mathcal{K}_{\beta}(k ; \varkappa): \widetilde{e}(k+2 \pi N) \in \widehat{S} \widetilde{\Omega}_{\lambda}\right\}, \\
\mathcal{K}_{\beta}^{\lambda}=\mathcal{K}_{\beta}^{\lambda}(k, \varkappa ; \phi)=\left\{N \in \mathcal{K}_{\beta}(k ; \varkappa): \widetilde{e}(k+2 \pi N) \in \widehat{S} \Omega_{\lambda}\right\}, \quad \widetilde{\mathcal{K}}_{\beta}^{\lambda} \subset \mathcal{K}_{\beta}^{\lambda} .
\end{gathered}
$$

The choice of the orthogonal transformation $\widehat{S}$ means that

$$
\left\|\left(\widehat{P}^{ \pm} \phi\right)^{\mathcal{K}_{\beta} \backslash \bigcup_{\lambda} \widetilde{\mathcal{K}}_{\beta}^{\lambda}}\right\|^{2} \leq \widetilde{\varepsilon}\left\|\widehat{P}^{ \pm} \phi\right\|^{2}
$$

For each index $\lambda$ (and for all already chosen $k, \boldsymbol{\varkappa}$, and $\phi$ ), we take an orthogonal system of vectors $\mathcal{E}_{j}^{(\lambda)} \in S_{n-1}, j=1, \ldots, n$, such that $\mathcal{E}_{1}^{(\lambda)}=$ $\tilde{e}^{\lambda}$ and $\mathcal{E}_{2}^{(\lambda)}=\mathcal{E}_{2}=e$. We let $x_{j}^{(\lambda)}=\left(x, \mathcal{E}_{j}^{(\lambda)}\right)$ denote the coordinates of the vectors $x=\sum_{j=1}^{n} x_{j} \mathcal{E}_{j} \in \mathbb{R}^{n}$ (and also of the vectors in $\mathbb{C}^{n}$ ). Let $\mathcal{E}_{j}^{(\lambda)}=\sum_{l=1}^{n} T_{l j}^{(\lambda)} \mathcal{E}_{l}$. Then $A_{j}^{(\lambda)}=\sum_{l=1}^{n} T_{l j}^{(\lambda)} A_{l}\left(\right.$ where $A_{l}=\left(A, \mathcal{E}_{l}\right)$ and $A_{j}^{(\lambda)}=$ $\left.\left(A, \mathcal{E}_{j}^{(\lambda)}\right)\right), \widetilde{A}_{j}^{(\lambda)}=\widetilde{A}_{j}^{(\lambda)}\left(\gamma, \mu, \widetilde{e}^{\lambda} ;.\right)=\sum_{l=1}^{n} T_{l j}^{(\lambda)} \widetilde{A}_{l}$, and $\widetilde{A}_{l}=\widetilde{A}_{l}\left(\gamma, \mu, \widetilde{e}^{\lambda} ;.\right)$. We introduce the notation $\widehat{\alpha}_{j}^{(\lambda)}=\sum_{l=1}^{n} T_{l j}^{(\lambda)} \widehat{\alpha}_{l}, j=1, \ldots, n$. For the Fourier coefficients $\left(\widetilde{A}_{j}^{(\lambda)}\right)_{N}$ of the functions $\widetilde{A}_{j}^{(\lambda)}, j=1, \ldots, n$, we have $\left(\widetilde{A}_{j}^{(\lambda)}\right)_{N}=$ 
$\widehat{\mu}\left(2 \pi N_{1}^{(\lambda)}\right)\left(A_{j}^{(\lambda)}\right)_{N}$ if $N_{2}=0$ and $\left(\widetilde{A}_{j}^{(\lambda)}\right)_{N}=0$ if $N_{2} \neq 0$. (Here, $\left(A_{j}^{(\lambda)}\right)_{N}$ are the Fourier coefficients of $A_{j}^{(\lambda)}, N \in \Lambda^{*}$.)

Let $\Phi^{(s, \lambda)}: \mathbb{R}^{n} \rightarrow \mathbb{C}, s=1,2$, be periodic trigonometric polynomials with the period lattice $\Lambda$ and the Fourier coefficients $\Phi_{N}^{(1, \lambda)}=\Phi_{N}^{(2, \lambda)}=0$ if $N_{1}^{(\lambda)}=N_{2}=0$ and

$$
\begin{gathered}
\Phi_{N}^{(1, \lambda)}=\left(2 \pi i\left(\left(N_{1}^{(\lambda)}\right)^{2}+N_{2}^{2}\right)\right)^{-1}\left(N_{1}^{(\lambda)}\left(A_{1}^{(\lambda)}-\widetilde{A}_{1}^{(\lambda)}\right)_{N}+N_{2}\left(A_{2}-\widetilde{A}_{2}\right)_{N}\right), \\
\Phi_{N}^{(2, \lambda)}=-\left(2 \pi i\left(\left(N_{1}^{(\lambda)}\right)^{2}+N_{2}^{2}\right)\right)^{-1}\left(N_{2}\left(A_{1}^{(\lambda)}-\widetilde{A}_{1}^{(\lambda)}\right)_{N}-N_{1}^{(\lambda)}\left(A_{2}-\widetilde{A}_{2}\right)_{N}\right)
\end{gathered}
$$

otherwise. We have

$$
\frac{\partial \Phi^{(1, \lambda)}}{\partial x_{1}^{(\lambda)}}-\frac{\partial \Phi^{(2, \lambda)}}{\partial x_{2}}=A_{1}^{(\lambda)}-\widetilde{A}_{1}^{(\lambda)}, \quad \frac{\partial \Phi^{(1, \lambda)}}{\partial x_{2}}+\frac{\partial \Phi^{(2, \lambda)}}{\partial x_{1}^{(\lambda)}}=A_{2}-\widetilde{A}_{2} .
$$

Lemma 1. There is a universal constant $C>0$ such that

$$
\left\|\Phi^{(s, \lambda)}\right\|_{L^{\infty}\left(\mathbb{R}^{n}\right)} \leq C\|\mu\| \max \left\{|\gamma|, h^{-1}\right\}\|A\|_{L^{\infty}\left(\mathbb{R}^{n} ; \mathbb{C}^{n}\right)}, \quad s=1,2 .
$$

Proof. Let $\eta(.) \in C^{\infty}(\mathbb{R} ; \mathbb{R}), \eta(\tau)=0$ for $\tau \leq \pi, 0 \leq \eta(\tau) \leq 1$ for $\pi<\tau \leq 2 \pi$, and $\eta(\tau)=1$ for $\tau>2 \pi$. For $x, y \in \mathbb{R}$ (and $x^{2}+y^{2}>0$ ), we set

$$
G(x, y)=\frac{x}{x^{2}+y^{2}} \int_{0}^{+\infty} \frac{\partial \eta(\tau)}{\partial \tau} J_{0}\left(\tau \sqrt{x^{2}+y^{2}}\right) d \tau,
$$

where $J_{0}($.$) is the Bessel function of the first kind of order zero; G(.,.) \in$ $L^{q}\left(\mathbb{R}^{2}\right), q \in[1,2)$. We write $G_{1}(t ; x, y)=t^{-1} G\left(t^{-1} x, t^{-1} y\right), t>0$, and $G_{2}(t ; x, y)=G_{1}(t ; y, x) ;\left\|G_{s}(t ; ., .)\right\|_{L^{1}\left(\mathbb{R}^{2}\right)}=t\|G(., .)\|_{L^{1}\left(\mathbb{R}^{2}\right)}, s=1,2$. For arbitrary continuous periodic functions $\mathcal{F}: \mathbb{R}^{n} \rightarrow \mathbb{C}$ with the period lattice $\Lambda$, we set

$$
\left(\mathcal{F} *_{\lambda} G_{s}(t ; ., .)\right)(x)=\iint_{\mathbb{R}^{2}} G_{s}\left(t ; \xi_{1}, \xi_{2}\right) \mathcal{F}\left(x-\xi_{1} \widetilde{e}^{\lambda}-\xi_{2} e\right) d \xi_{1} d \xi_{2}, \quad x \in \mathbb{R}^{n} .
$$

In this case, $\left(\mathcal{F} *_{\lambda} G_{s}(t ; ., .)\right)_{N}=0$ if $N_{1}^{(\lambda)}=N_{2}=0$ and

$$
\left(\mathcal{F} *_{\lambda} G_{s}(t ; ., .)\right)_{N}=-\frac{i N_{s}^{(\lambda)}}{\left(N_{1}^{(\lambda)}\right)^{2}+N_{2}^{2}} \eta\left(2 \pi t \sqrt{\left(N_{1}^{(\lambda)}\right)^{2}+N_{2}^{2}}\right) \mathcal{F}_{N}
$$

otherwise, $s=1,2$. Let $t=\max \left\{|\gamma|, h^{-1}\right\}$. Because $(A-\widetilde{A})_{N}=0$ for $N_{2}=0,\left|N_{1}^{(\lambda)}\right| \leq h$, and $\left|N_{2}\right|=|\gamma|^{-1}|(N, \gamma)| \geq|\gamma|^{-1}$ for $N_{2} \neq 0$, we have

$$
2 \pi \Phi^{(1, \lambda)}=\left(A_{1}^{(\lambda)}-\widetilde{A}_{1}^{(\lambda)}\right) *_{\lambda} G_{1}(t ; . . .)+\left(A_{2}-\widetilde{A}_{2}\right) *_{\lambda} G_{2}(t ; . . .),
$$




$$
2 \pi \Phi^{(2, \lambda)}=-\left(A_{1}^{(\lambda)}-\widetilde{A}_{1}^{(\lambda)}\right) *_{\lambda} G_{2}(t ; . . .)+\left(A_{2}-\widetilde{A}_{2}\right) *_{\lambda} G_{1}(t ; ., .) .
$$

Using the inequalities $\|\widetilde{A}\|_{L^{\infty}\left(\mathbb{R}^{n} ; \mathbb{C}^{n}\right)} \leq\|\mu\|\|A\|_{L^{\infty}\left(\mathbb{R}^{n} ; \mathbb{C}^{n}\right)}$ and $\|\mu\| \geq 1$, and taking the constant $C=2 \pi^{-1}\|G(., .)\|_{L^{1}\left(\mathbb{R}^{2}\right)}$, we complete the proof of the lemma.

We introduce the notation

$$
\begin{gathered}
\widehat{D}_{0}^{(\lambda)}=\left(-i \frac{\partial}{\partial x_{1}^{(\lambda)}}+k_{1}^{(\lambda)}\right) \widehat{\alpha}_{1}^{(\lambda)}+\left(-i \frac{\partial}{\partial x_{2}}+k_{2}+i \varkappa\right) \widehat{\alpha}_{2}, \\
\widehat{D}^{(\lambda)}=\widehat{D}_{0}^{(\lambda)}-\widetilde{A}_{1}^{(\lambda)} \widehat{\alpha}_{1}^{(\lambda)}-\widetilde{A}_{2} \widehat{\alpha}_{2}, \\
\widehat{D}^{(\lambda)}(k+i \varkappa e)=e^{-i \widehat{\alpha}_{1}^{(\lambda)} \widehat{\alpha}_{2} \Phi^{(2, \lambda)}} e^{i \Phi^{(1, \lambda)}} \widehat{D}^{(\lambda)} e^{-i \Phi^{(1, \lambda)}} e^{-i \widehat{\alpha}_{1}^{(\lambda)} \widehat{\alpha}_{2} \Phi^{(2, \lambda)}}, \\
\widehat{\mathcal{V}}^{(\lambda)}=\widehat{V}^{(0)}+\widehat{V}^{(1)}+\sum_{j=3}^{n}\left(-i \frac{\partial}{\partial x_{j}^{(\lambda)}}+k_{j}^{(\lambda)}-A_{j}^{(\lambda)}\right) \widehat{\alpha}_{j}^{(\lambda)}, \\
\widehat{D}(k+i \varkappa e)=\widehat{D}^{(\lambda)}(k+i \varkappa e)+\widehat{\mathcal{V}}^{(\lambda)} .
\end{gathered}
$$

If $N \in \mathcal{K}_{\beta}^{\lambda}$, then $\left|\widetilde{e}(k+2 \pi N)-\widetilde{e}^{\lambda}\right|<\rho$ and therefore

$$
\left|k+2 \pi N-\left(k_{2}+2 \pi N_{2}\right) e-\varkappa \widetilde{e}^{\lambda}\right|<\beta+\rho \varkappa .
$$

It follows that

$$
\left|\sum_{j=3}^{n}\left(k_{j}^{(\lambda)}+2 \pi N_{j}^{(\lambda)}\right) \mathcal{E}_{j}^{(\lambda)}\right|<\beta+\rho \varkappa, \quad\left|k_{1}^{(\lambda)}+2 \pi N_{1}^{(\lambda)}-\varkappa\right|<\beta+\rho \varkappa,
$$

and

$$
\left\|\widehat{\mathcal{V}}^{(\lambda)} \phi^{\mathcal{K}_{\beta}^{\lambda}}\right\| \leq\left(\beta+\left(c_{6}+2\right) R+W\right)\left\|\phi^{\mathcal{K}_{\beta}^{\lambda}}\right\| .
$$

We use the brief notation $\widehat{P}_{\lambda}^{ \pm}=\widehat{P}_{\widetilde{e}^{\lambda}}^{ \pm}=(1 / 2)\left(\widehat{I} \pm i \widehat{\alpha}_{1}^{(\lambda)} \widehat{\alpha}_{2}\right)$. We set $\chi^{(\lambda)}=$ $e^{-i \Phi^{(1, \lambda)}} e^{-i \widehat{\alpha}_{1}^{(\lambda)} \widehat{\alpha}_{2} \Phi^{(2, \lambda)}} \phi^{\mathcal{K}_{\beta}^{\lambda}}$. The relation

$$
\left.\widehat{D}_{0}^{(\lambda)} \widehat{P}_{\lambda}^{ \pm} \chi^{(\lambda)}=\sum_{N \in \Lambda^{*}}\left(k_{2}+2 \pi N_{2}+i\left(k_{1}^{(\lambda)}+2 \pi N_{1}^{(\lambda)}\right)\right)\right) \widehat{\alpha}_{2} \widehat{P}_{\lambda}^{ \pm} \chi_{N}^{(\lambda)} e^{2 \pi i(N, x)}
$$

holds.

We write $\mathcal{O}^{(\lambda)}(\tau)=\left\{N \in \Lambda^{*}:\left|k_{1}^{(\lambda)}+2 \pi N_{1}^{(\lambda)}-\varkappa\right|<2 \tau\right\}, \tau>0$. Inequalities (9) imply that there is a constant

$$
c_{7}=c_{7}\left(\tilde{\theta}, \theta, W_{0}, R, \beta\right)>\frac{1}{2}\left(\beta+\left(c_{6}+2\right) R\right)
$$

such that (for all $\lambda$ )

$$
\left\|\sum_{N \in \Lambda^{*} \backslash \mathcal{O}^{(\lambda)}\left(c_{7}\right)} \widehat{P}_{\lambda}^{+} \chi_{N}^{(\lambda)} e^{2 \pi i(N, x)}\right\| \leq \frac{1}{2}\left\|\widehat{P}_{\lambda}^{+} \chi^{(\lambda)}\right\| .
$$


In what follows, we assume that $\varkappa_{0} \geq c_{7}$. As a consequence of (10) and (11), we obtain

$$
\begin{aligned}
& \left\|\widehat{D}_{0}^{(\lambda)} \widehat{P}_{\lambda}^{+} \chi^{(\lambda)}\right\| \geq v^{1 / 2}(K)\left(\sum_{N \in \mathcal{O}^{(\lambda)}\left(c_{7}\right)}\left|\varkappa+\left(k_{1}^{(\lambda)}+2 \pi N_{1}^{(\lambda)}\right)\right|^{2}\left\|\widehat{P}_{\lambda}^{+} \chi_{N}^{(\lambda)}\right\|^{2}\right)^{1 / 2} \geq \\
& \quad 2\left(\varkappa-c_{7}\right)\left\|\sum_{N \in \mathcal{O}^{(\lambda)}\left(c_{7}\right)} \widehat{P}_{\lambda}^{+} \chi_{N}^{(\lambda)} e^{2 \pi i(N, x)}\right\| \geq\left(\varkappa-c_{7}\right)\left\|\widehat{P}_{\lambda}^{+} \chi^{(\lambda)}\right\| .
\end{aligned}
$$

On the other hand, we have $\left|k_{2}+2 \pi N_{2}\right| \geq \pi|\gamma|^{-1}$. Condition (6) implies that

$$
\left\|\widetilde{A}_{1}^{(\lambda)} \widehat{\alpha}_{1}^{(\lambda)}+\widetilde{A}_{2} \widehat{\alpha}_{2}\right\|_{L^{\infty}\left(\mathbb{R}^{n} ; \mathcal{L}_{M}\right)} \leq \widetilde{\theta} \pi|\gamma|^{-1}
$$

and therefore (see (10))

$\left\|\widehat{D}^{(\lambda)} \widehat{P}_{\lambda}^{-} \chi^{(\lambda)}\right\| \geq\left\|\widehat{D}_{0}^{(\lambda)} \widehat{P}_{\lambda}^{-} \chi^{(\lambda)}\right\|-\widetilde{\theta} \pi|\gamma|^{-1}\left\|\widehat{P}_{\lambda}^{-} \chi^{(\lambda)}\right\| \geq(1-\widetilde{\theta}) \pi|\gamma|^{-1}\left\|\widehat{P}_{\lambda}^{-} \chi^{(\lambda)}\right\|$.

The operators $\widehat{P}_{\lambda}^{ \pm}$commute with the operators $e^{ \pm i \Phi^{(1, \lambda)}}, e^{-i \widehat{\alpha}_{1}^{(\lambda)} \widehat{\alpha}_{2} \Phi^{(2, \lambda)}}$, and $\widehat{\mathcal{V}}^{(\lambda)}$, and we have $\widehat{P}_{\lambda}^{ \pm} \widehat{D}^{(\lambda)}=\widehat{D}^{(\lambda)} \widehat{P}_{\lambda}^{\mp}$. Consequently,

$$
\widehat{P}_{\lambda}^{ \pm} \widehat{D}(k+i \varkappa e)=\widehat{D}^{(\lambda)}(k+i \varkappa e) \widehat{P}_{\lambda}^{\mp}+\widehat{\mathcal{V}}^{(\lambda)} \widehat{P}_{\lambda}^{ \pm} .
$$

Using the above estimates and also the inequality

$$
\left\|e^{ \pm i \Phi^{(1, \lambda)}} e^{i \widehat{\alpha}_{1}^{(\lambda)} \widehat{\alpha}_{2} \Phi^{(2, \lambda)}}\right\|_{L^{\infty}\left(\mathbb{R}^{n} ; \mathcal{L}_{M}\right)} \leq c_{5}^{-1 / 2}(A),
$$

we derive

$$
\begin{gathered}
\left\|\widehat{P}_{\lambda}^{+} \widehat{D}(k+i \varkappa e) \phi \mathcal{K}_{\beta}^{\lambda}\right\| \geq(1-\widetilde{\theta}) \pi|\gamma|^{-1} c_{5}(A)\left\|\widehat{P}_{\lambda}^{-} \phi^{\mathcal{K}_{\beta}^{\lambda}}\right\|-\left\|\widehat{\mathcal{V}}^{(\lambda)} \widehat{P}_{\lambda}^{+} \phi^{\mathcal{K}_{\beta}^{\lambda}}\right\|, \\
\left\|\widehat{P}_{\lambda}^{-} \widehat{D}(k+i \varkappa e) \phi^{\mathcal{K}_{\beta}^{\lambda}}\right\| \geq \\
\left(\varkappa-c_{7}-\pi|\gamma|^{-1}\right) c_{5}(A)\left\|\widehat{P}_{\lambda}^{+} \phi \mathcal{K}_{\beta}^{\lambda}\right\|-\left\|\widehat{\mathcal{V}}^{(\lambda)} \widehat{P}_{\lambda}^{-} \phi^{\mathcal{K}_{\beta}^{\lambda}}\right\| .
\end{gathered}
$$

Let

$$
\begin{gathered}
\sigma=\theta_{2}^{2} \theta_{3}^{-2}-1, \\
\widetilde{a}=\min \left\{1, \sqrt{\sigma} a,\left(1-\tilde{\theta}-\theta_{1}\right) \pi|\gamma|^{-1} c_{5}^{\prime}\left(\beta+\left(c_{6}+2\right) R+W_{0}\right)^{-1}\right\}, \\
b^{\prime \prime}=\min \left\{\begin{array}{l}
1, \\
\sqrt{\sigma} a, \\
\left(1-\tilde{\theta}-\theta_{1}\right) \pi|\gamma|^{-1} c_{5}^{\prime}\left(c_{6}+2\right)^{-1}\left(1+W_{0}\right)^{-1}, \\
2\left(\theta_{1}-\theta_{2}\right) \pi|\gamma|^{-1}\left(c_{6}+2\right)^{-1} .
\end{array}\right.
\end{gathered}
$$


Since $(\beta+R)^{-1} b^{\prime \prime}<\widetilde{a}$, we can pick a number $\widetilde{a}^{\prime}$ such that $(\beta+R)^{-1} b^{\prime \prime} \leq$ $\widetilde{a}^{\prime}<\widetilde{a}$. For an adequate choice of the number $\varkappa_{0}$ (and for $\varkappa \geq \varkappa_{0}$ ), inequalities (12) and (13) imply the estimate

$$
\begin{gathered}
\left\|\widehat{P}_{\lambda}^{+} \widehat{D}(k+i \varkappa e) \phi^{\mathcal{K}_{\beta}^{\lambda}}\right\|+\widetilde{a}\left\|\widehat{P}_{\lambda}^{-} \widehat{D}(k+i \varkappa e) \phi^{\mathcal{K}_{\beta}^{\lambda}}\right\| \geq \\
c_{5}(A)\left(\theta_{1} \pi|\gamma|^{-1}\left\|\widehat{P}_{\lambda}^{-} \phi^{\mathcal{K}_{\beta}^{\lambda}}\right\|+\widetilde{a}^{\prime} \varkappa\left\|\widehat{P}_{\lambda}^{+} \phi^{\mathcal{K}_{\beta}^{\lambda}}\right\|\right) .
\end{gathered}
$$

For all $\widetilde{e} \in \widehat{S} \Omega_{\lambda} \subset S_{n-2}(e)$, we have

$$
\left\|\left(\widehat{P}^{ \pm}-\widehat{P}_{\lambda}^{ \pm}\right) \phi^{\mathcal{K}_{\beta}^{\lambda}}\right\| \leq \frac{1}{2}\left|\widetilde{e}-\widetilde{e}^{\lambda}\right|\left\|\phi^{\mathcal{K}_{\beta}^{\lambda}}\right\| \leq \frac{\rho}{2}\left\|\phi^{\mathcal{K}_{\beta}^{\lambda}}\right\| .
$$

If $\left(\widehat{D}(k+i \varkappa e) \phi^{\mathcal{K}_{\beta}^{\lambda}}\right)_{N} \neq 0$ for some $N \in \Lambda^{*}$, then $k+2 \pi N \notin \mathcal{P}(e)$ and $\left|\widetilde{e}(k+2 \pi N)-\widetilde{e}^{\lambda}\right|<\rho+2 R / \varkappa=\rho^{\prime}$. Therefore,

$$
\left\|\left(\widehat{P}^{ \pm}-\widehat{P}_{\lambda}^{ \pm}\right) \widehat{D}(k+i \varkappa e) \phi^{\mathcal{K}_{\beta}^{\lambda}}\right\| \leq \frac{\rho^{\prime}}{2}\left\|\widehat{D}(k+i \varkappa e) \phi^{\mathcal{K}_{\beta}^{\lambda}}\right\| .
$$

Consequently,

$$
\begin{gathered}
\left\|\widehat{P}_{\lambda}^{+} \widehat{D}(k+i \varkappa e) \phi^{\mathcal{K}_{\beta}^{\lambda}}\right\|+\widetilde{a}\left\|\widehat{P}_{\lambda}^{-} \widehat{D}(k+i \varkappa e) \phi^{\mathcal{K}_{\beta}^{\lambda}}\right\| \leq \\
\left(1+\rho^{\prime} \widetilde{a}^{-1}\right)\left(\left\|\widehat{P}^{+} \widehat{D}(k+i \varkappa e) \phi^{\mathcal{K}_{\beta}^{\lambda}}\right\|+\widetilde{a}\left\|\widehat{P}^{-} \widehat{D}(k+i \varkappa e) \phi^{\mathcal{K}_{\beta}^{\lambda}}\right\|\right) .
\end{gathered}
$$

Since $(\beta+R)^{-1} b^{\prime \prime} \leq \widetilde{a}^{\prime}$ and $\left(c_{6}+2\right) b^{\prime \prime} \leq 2\left(\theta_{1}-\theta_{2}\right) \pi|\gamma|^{-1}$, for an adequately chosen number $\varkappa_{0}$ (and for $\varkappa \geq \varkappa_{0}$ ) inequality (14) implies that

$$
\begin{gathered}
\theta_{1} \frac{\pi}{|\gamma|}\left\|\widehat{P}_{\lambda}^{-} \phi^{\mathcal{K}_{\beta}^{\lambda}}\right\|+\frac{b^{\prime \prime} \varkappa}{\beta+R}\left\|\widehat{P}_{\lambda}^{+} \phi^{\mathcal{K}_{\beta}^{\lambda}}\right\| \geq \\
\left(1+\rho^{\prime} \widetilde{a}^{-1}\right)\left(\theta_{2} \frac{\pi}{|\gamma|}\left\|\widehat{P}^{-} \phi^{\mathcal{K}_{\beta}^{\lambda}}\right\|+\frac{b^{\prime \prime} \varkappa}{2(\beta+R)}\left\|\widehat{P}^{+} \phi^{\mathcal{K}_{\beta}^{\lambda}}\right\|\right) .
\end{gathered}
$$

From (15) and (16), it follows that

$$
\begin{gathered}
\left\|\widehat{P}^{+} \widehat{D}(k+i \varkappa e) \phi^{\mathcal{K}_{\beta}^{\lambda}}\right\|+\widetilde{a}\left\|\widehat{P}^{-} \widehat{D}(k+i \varkappa e) \phi^{\mathcal{K}_{\beta}^{\lambda}}\right\| \geq \\
c_{5}(A)\left(\theta_{2} \frac{\pi}{|\gamma|}\left\|\widehat{P}^{-} \phi^{\mathcal{K}_{\beta}^{\lambda}}\right\|+\frac{b^{\prime \prime} \varkappa}{2(\beta+R)}\left\|\widehat{P}^{+} \phi^{\mathcal{K}_{\beta}^{\lambda}}\right\|\right) .
\end{gathered}
$$

We write $b^{\prime}=(1 / 2)(1+\sigma)^{-1 / 2} b^{\prime \prime}$. Then

$$
\begin{gathered}
\left\|\widehat{P}^{+} \widehat{D}(k+i \varkappa e) \phi^{\mathcal{K}_{\beta}^{\lambda}}\right\|^{2}+a^{2}\left\|\widehat{P}^{-} \widehat{D}(k+i \varkappa e) \phi^{\mathcal{K}_{\beta}^{\lambda}}\right\|^{2} \geq \\
(1+\sigma)^{-1}\left(\left\|\widehat{P}^{+} \widehat{D}(k+i \varkappa e) \phi^{\mathcal{K}_{\beta}^{\lambda}}\right\|+\widetilde{a}\left\|\widehat{P}^{-} \widehat{D}(k+i \varkappa e) \phi^{\mathcal{K}_{\beta}^{\lambda}}\right\|\right)^{2} \geq \\
c_{5}^{2}(A)\left(\left(\theta_{3} \frac{\pi}{|\gamma|}\right)^{2}\left\|\widehat{P}^{-} \phi^{\mathcal{K}_{\beta}^{\lambda}}\right\|^{2}+\left(\frac{b^{\prime} \varkappa}{2(\beta+R)}\right)^{2}\left\|\widehat{P}^{+} \phi^{\mathcal{K}_{\beta}^{\lambda}}\right\|^{2}\right) .
\end{gathered}
$$


If $N \in \Lambda^{*}$ and $\lambda_{1} \neq \lambda_{2}$, then either $2 \pi\left|N-N^{\prime}\right|>R$ for all $N^{\prime} \in \mathcal{K}_{\beta}^{\lambda_{1}}$ or $2 \pi\left|N-N^{\prime \prime}\right|>R$ for all $N^{\prime \prime} \in \mathcal{K}_{\beta}^{\lambda_{2}}$. Therefore,

$$
\widehat{V} \phi_{\lambda}^{\cup \mathcal{K}_{\beta}^{\lambda}}=\sum_{\lambda} \widehat{V} \phi^{\mathcal{K}_{\beta}^{\lambda}}, \quad \widehat{D}(k+i \varkappa e) \phi^{\cup} \mathcal{K}_{\beta}^{\lambda}=\sum_{\lambda} \widehat{D}(k+i \varkappa e) \phi^{\mathcal{K}_{\beta}^{\lambda}} .
$$

If $N \in \bigcup_{\lambda} \mathcal{K}_{\beta}^{\lambda}$, then

$$
(\widehat{D}(k+i \varkappa e) \phi)_{N}=\left(\widehat{D}(k+i \varkappa e) \phi \bigcup^{\bigcup \mathcal{K}_{\beta}^{\lambda}}\right)_{N}+\left(\widehat{V} \phi^{\mathcal{K}_{\beta} \backslash \bigcup_{\lambda} \mathcal{K}_{\beta}^{\lambda}}\right)_{N} .
$$

If $N \in \Lambda^{*} \backslash \bigcup_{\lambda} \mathcal{K}_{\beta}^{\lambda}$, then

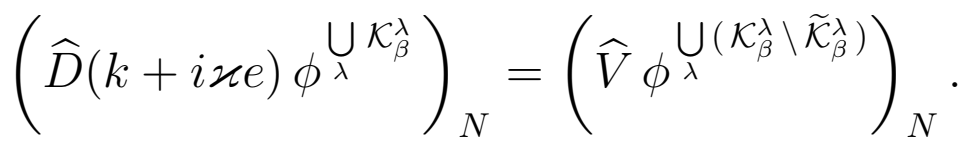

These relations (for each of the signs) imply the estimates

$$
\begin{aligned}
& \left\|\widehat{P}^{ \pm} \widehat{D}(k+i \varkappa e) \phi\right\|^{2} \geq \\
& v(K) \sum_{N \in \bigcup_{\lambda} \mathcal{K}_{\beta}^{\lambda}}\left\|\left(\widehat{P}^{ \pm} \widehat{D}(k+i \varkappa e) \phi^{\bigcup} \mathcal{K}_{\beta}^{\lambda}\right)_{N}+\left(\widehat{P}^{ \pm} \widehat{V} \phi^{\mathcal{K}_{\beta} \backslash \bigcup_{\lambda} \mathcal{K}_{\beta}^{\lambda}}\right)_{N}\right\| \geq \\
& (1-\delta)\left\|\widehat{P}^{ \pm} \widehat{D}(k+i \varkappa e) \phi_{\lambda}^{\cup} \mathcal{K}_{\beta}^{\lambda}\right\|^{2}- \\
& (1-\delta)\left\|\widehat{P}^{\Lambda^{*} \backslash \bigcup_{\lambda} \mathcal{K}_{\beta}^{\lambda}} \widehat{P}^{ \pm} \widehat{V} \phi^{\bigcup} \bigcup_{\beta}^{\left(\mathcal{K}_{\beta}^{\lambda} \backslash \widetilde{\mathcal{K}}_{\beta}^{\lambda}\right)}\right\|^{2}- \\
& (1-\delta) \delta^{-1}\left\|\widehat{P}_{\lambda}^{\bigcup \mathcal{K}_{\beta}^{\lambda}} \widehat{P}^{ \pm} \widehat{V} \phi \mathcal{K}_{\beta} \backslash \bigcup_{\lambda} \mathcal{K}_{\beta}^{\lambda}\right\|^{2} \geq \\
& (1-\delta)\left\|\widehat{P}^{ \pm} \widehat{D}(k+i \varkappa e) \phi^{\bigcup} \mathcal{K}_{\beta}^{\lambda}\right\|^{2}-\left(1-\delta^{2}\right) \delta^{-1} W^{2}\left\|\phi^{\mathcal{K}_{\beta} \backslash \bigcup_{\lambda} \mathcal{K}_{\beta}^{\lambda}}\right\|^{2} \geq \\
& (1-\delta)\left\|\widehat{P}^{ \pm} \widehat{D}(k+i \varkappa e) \phi \bigcup_{\lambda} \mathcal{K}_{\beta}^{\lambda}\right\|^{2}-\widetilde{\varepsilon} \delta^{-1} W^{2}\|\phi\|^{2} .
\end{aligned}
$$

We set $b=(1 / 4) \sqrt{(1-\delta)(1-\widetilde{\varepsilon})} b^{\prime}$. For $\varkappa_{0}$, we assume that $3\left(c_{5}^{\prime}\right)^{2} b^{2} \varkappa_{0}^{2} \geq$ $8 \widetilde{\varepsilon} \delta^{-1} W_{0}^{2} R^{2}$. Then for $\varkappa \geq \varkappa_{0}$, from (17) and (18) (in view of (8) and the constraint $a \leq 1$ ) we obtain the inequalities

$$
\begin{gathered}
\left\|\widehat{P}^{+} \widehat{D}(k+i \varkappa e) \phi\right\|^{2}+a^{2}\left\|\widehat{P}^{-} \widehat{D}(k+i \varkappa e) \phi\right\|^{2} \geq \\
(1-\delta) c_{5}^{2}(A) \sum_{\lambda}\left(\left(\theta_{3} \frac{\pi}{|\gamma|}\right)^{2}\left\|\widehat{P}^{-} \phi^{\mathcal{K}_{\beta}^{\lambda}}\right\|^{2}+\left(\frac{b^{\prime} \varkappa}{2(\beta+R)}\right)^{2}\left\|\widehat{P}^{+} \phi^{\mathcal{K}_{\beta}^{\lambda}}\right\|^{2}\right)-
\end{gathered}
$$




$$
\begin{gathered}
\frac{2}{\delta} W^{2} \widetilde{\varepsilon}\|\phi\|^{2} \geq \\
(1-\widetilde{\varepsilon}) c_{5}^{2}(A)\left(\left(\theta_{4} \frac{\pi}{|\gamma|}\right)^{2}\left\|\widehat{P}^{-} \phi\right\|^{2}+(1-\delta)\left(\frac{b^{\prime} \varkappa}{2(\beta+R)}\right)^{2}\left\|\widehat{P}^{+} \phi\right\|^{2}\right)- \\
\frac{2}{\delta} W^{2} \widetilde{\varepsilon}\left(\left\|\widehat{P}^{-} \phi\right\|^{2}+\left\|\widehat{P}^{+} \phi\right\|^{2}\right) \geq \\
c_{5}^{2}(A)\left(\left(\theta \frac{\pi}{|\gamma|}\right)^{2}\left\|\widehat{P}^{-} \phi\right\|^{2}+(1-\delta)\left(\frac{b \varkappa}{\beta+R}\right)^{2}\left\|\widehat{P}^{+} \phi\right\|^{2}\right) .
\end{gathered}
$$

Theorem 5 is proved.

3. The following theorems are a consequence of Theorem 5 . The proof of Theorem 6 is based on applying the relation

$$
\widehat{P}^{ \pm}(k) \widehat{D}_{0}(k+i \varkappa e)=\widehat{D}_{0}(k+i \varkappa e) \widehat{P}^{\mp}(k)
$$

and on selecting an arbitrarily small number $a \in(0,1]$. The proof of Theorem 7 essentially uses the arbitrariness in the choice of the number $\beta>0$ (see below). Theorem 6 is used to prove the absolute continuity of the spectrum of a periodic Schrödinger operator.

Theorem 6. Let $\tilde{\theta} \in[0,1), W_{0} \geq 0, R \geq 1$, and $\beta>0$ (for a fixed vector $\gamma \in \Lambda \backslash\{0\}$ and a fixed measure $\left.\mu \in \mathcal{M}_{h}, h>0 ; e=|\gamma|^{-1} \gamma\right)$. Then there are numbers $c_{8}=c_{8}\left(\widetilde{\theta}, W_{0}\right)>0$ and $\varkappa_{0}=\varkappa_{0}\left(\widetilde{\theta}, W_{0}, R, \beta\right)>4 \beta+5 R$ such that for all vectors $k \in \mathbb{R}^{n}$ with $(k, \gamma)=\pi$, all $\varkappa \geq \varkappa_{0}$, all continuous periodic functions $\widehat{V}^{(s)}: \mathbb{R}^{n} \rightarrow \mathcal{L}_{M}^{(s)}, s=0,1$, and $A: \mathbb{R}^{n} \rightarrow \mathbb{C}^{n}$ (with the period lattice $\left.\Lambda \subset \mathbb{R}^{n}, n \geq 3\right)$ for which $A_{0}=0$ and conditions (5) - (7) are satisfied, and all vector functions $\phi \in \mathcal{H}\left(\mathcal{K}_{\beta}(k ; \varkappa)\right)$, the inequality

$$
\left\|\widehat{D}^{2}(k+i \varkappa e) \phi\right\| \geq \frac{c_{8} \varkappa}{\beta+R}\|\phi\|
$$

holds.

Theorem 7. Let $\tilde{\theta} \in[0,1), \theta \in(0,1-\widetilde{\theta}), W_{0} \geq 0, R \geq 1$, and $\delta \in(0,1]$ (for a fixed vector $\gamma \in \Lambda \backslash\{0\}$ and a fixed measure $\mu \in \mathcal{M}_{h}$, $\left.h>0 ; e=|\gamma|^{-1} \gamma\right)$. Then there are numbers $\mathcal{D}=\mathcal{D}\left(\theta, W_{0}, \delta\right) \geq 1$ and $\varkappa_{0}=\varkappa_{0}\left(\tilde{\theta}, \theta, W_{0}, R, \delta\right)>(4 \mathcal{D}+1) R$ such that for all vectors $k \in \mathbb{R}^{n}$ with $(k, \gamma)=\pi$, all $\varkappa \geq \varkappa_{0}$, all continuous periodic functions $\widehat{V}^{(s)}: \mathbb{R}^{n} \rightarrow \mathcal{L}_{M}^{(s)}$, $s=0,1$, and $A: \mathbb{R}^{n} \rightarrow \mathbb{C}^{n}$ (with the period lattice $\Lambda \subset \mathbb{R}^{n}, n \geq 3$ ) for which $A_{0}=0$ and conditions (5) - (7) are satisfied, and all vector functions $\phi \in \widetilde{H}^{1}\left(K ; \mathbb{C}^{M}\right)$, the inequality

$$
\|\widehat{D}(k+i \varkappa e) \phi\|^{2} \geq
$$




$$
(1-\delta)\left(c_{5}^{2}(A)\left(\theta \frac{\pi}{|\gamma|}\right)^{2}\left\|\phi^{\mathcal{K}_{\mathcal{D} R}}\right\|^{2}+v(K) \sum_{N \in \Lambda^{*} \backslash \mathcal{K}_{\mathcal{D} R}} G_{N}^{2}(k ; \varkappa)\left\|\phi_{N}\right\|^{2}\right)
$$

holds.

Proof of Theorem 2. Let $\theta<\theta^{\prime}<1-\widetilde{\theta}, \widehat{V}_{\nu}^{(s)}: \mathbb{R}^{n} \rightarrow \mathcal{L}_{M}^{(s)}, s=0,1$, and $A_{\nu}: \mathbb{R}^{n} \rightarrow \mathbb{C}^{n}, \nu \in \mathbb{N}$, be sequences of trigonometric polynomials with the period lattice $\Lambda$ that uniformly converge as $\nu \rightarrow+\infty$ to the functions $\widehat{V}^{(s)}$ and $A$, let $\left(A_{\nu}\right)_{0}=0$ for all $\nu \in \mathbb{N}$, and let $\widehat{V}_{\nu}=\widehat{V}_{\nu}^{(0)}+\widehat{V}_{\nu}^{(1)}-\sum_{j=1}^{n}\left(A_{\nu}\right)_{j} \widehat{\alpha}_{j}$. From Theorem 7 (because $G_{N}(k ; \varkappa) \geq \pi|\gamma|^{-1}, N \in \Lambda^{*}$ ) it follows that for all sufficiently large $\nu$, there are numbers $\varkappa_{0}^{(\nu)}>0$ such that for all $k \in \mathbb{R}^{n}$ with $(k, \gamma)=\pi$, all $\varkappa \geq \varkappa_{0}^{(\nu)}$, and all vector functions $\phi \in \widetilde{H}^{1}\left(K ; \mathbb{C}^{M}\right)$, the inequality

$$
\left\|\left(\widehat{D}_{0}(k+i \varkappa e)+\widehat{V}_{\nu}\right) \phi\right\| \geq c_{5}\left(A_{\nu}\right) \theta^{\prime} \pi|\gamma|^{-1}\|\phi\|
$$

is valid. For a sufficiently large index $\nu$ (and for $\varkappa \geq \varkappa_{0}^{(\nu)}$ ), it follows that the desired inequality holds. Theorem 2 is proved.

Theorem 8. Let $\widehat{V}^{(s)}: \mathbb{R}^{n} \rightarrow \mathcal{L}_{M}^{(s)}, s=0,1$, and $A: \mathbb{R}^{n} \rightarrow \mathbb{C}^{n}$ be continuous periodic functions with the period lattice $\Lambda \subset \mathbb{R}^{n}, n \geq 3$. If $A_{0}=0$ and condition (6) with $\widetilde{\theta} \in[0,1)$ is satisfied for a vector $\gamma \in \Lambda \backslash\{0\}$ $\left(e=|\gamma|^{-1} \gamma\right)$ and a measure $\mu \in \mathcal{M}_{h}, h>0$, then for any $\delta \in(0,1]$, there are numbers $\beta=\beta(\gamma, h, \mu ; \widehat{V}, \delta)>0$ and $\varkappa_{0}=\varkappa_{0}(\gamma, h, \mu ; \widehat{V}, \delta)>0$ such that for all $k \in \mathbb{R}^{n}$ with $(k, \gamma)=\pi$, all $\varkappa \geq \varkappa_{0}$, and all vector functions $\phi \in \widetilde{H}^{1}\left(K ; \mathbb{C}^{M}\right)$, the inequality

$$
\begin{gathered}
\|\widehat{D}(k+i \varkappa e) \phi\|^{2} \geq(1-\delta)\left(c_{5}^{2}(A ; \gamma, h, \mu)(1-\widetilde{\theta})^{2}\left(\frac{\pi}{|\gamma|}\right)^{2}\left\|\phi^{\mathcal{K}_{\beta}}\right\|^{2}+\right. \\
\left.v(K) \sum_{N \in \Lambda^{*} \backslash \mathcal{K}_{\beta}} G_{N}^{2}(k ; \varkappa)\left\|\phi_{N}\right\|^{2}\right)
\end{gathered}
$$

holds.

Theorem 8 also follows from Theorem 7 in view of the uniform approximation of the functions $\widehat{V}^{(s)}$ and $A$ by trigonometric polynomials with the period lattice $\Lambda$.

Corollary. Let $\widehat{V}^{(s)}: \mathbb{R}^{n} \rightarrow \mathcal{L}_{M}^{(s)}, s=0,1$, and $A: \mathbb{R}^{n} \rightarrow \mathbb{C}^{n}$ be continuous periodic functions with the period lattice $\Lambda \subset \mathbb{R}^{n}, n \geq 3$, let $A_{0}=0$, and let condition (6) with $\widetilde{\theta} \in[0,1)$ hold for some vector $\gamma \in \Lambda \backslash\{0\}$ $\left(e=|\gamma|^{-1} \gamma\right)$ and a measure $\mu \in \mathcal{M}_{h}, h>0$. Then there are numbers $c_{9}=c_{9}(\gamma, h, \mu ; \widehat{V})>0$ and $\varkappa_{0}=\varkappa_{0}(\gamma, h, \mu ; \widehat{V})>0$ such that for all $k \in \mathbb{R}^{n}$ 
with $(k, \gamma)=\pi$, all $\varkappa \geq \varkappa_{0}$, and all vector functions $\phi \in \widetilde{H}^{1}\left(K ; \mathbb{C}^{M}\right)$, the inequality

$$
\|\widehat{D}(k+i \varkappa e) \phi\|^{2} \geq c_{9} v(K) \sum_{N \in \Lambda^{*}} G_{N}^{2}(k ; \varkappa)\left\|\phi_{N}\right\|^{2}
$$

is fulfilled.

\section{REFERENCES}

[1] M. Sh. Birman and T. A. Suslina, The periodic Dirac operator is absolutely continuous, Integral Equations and Operator Theory 34 (1999), 377-395.

[2] M. Sh. Birman and T. A. Suslina, Two-dimensional periodic magnetic Hamiltonian is absolutely continuous, Algebra i Analiz 9 (1997), no. 1, 32-48; English transl., St. Petersburg Math. J. 9 (1998), no. 1, 21-32.

[3] M. Sh. Birman and T. A. Suslina, Absolute continuity of the two-dimensional periodic magnetic Hamiltonian with discontinuous vector-valued potential, Algebra i Analiz 10 (1998), no. 4, 1-36; English transl., St. Petersburg Math. J. 10 (1999), no. 4, 579-601.

[4] L. I. Danilov, On the spectrum of the two-dimensional periodic Dirac operator, Teoret. Mat. Fiz. 118 (1999), no. 1, 3-14; English transl., Theoret. and Math. Phys. 118 (1999), no. 1, 1-11.

[5] L. I. Danilov, The spectrum of the Dirac operator with periodic potential: III [in Russian], Deposited at VINITI 10 July 1992, No. 2252-B92, VINITI, Moscow (1992).

[6] A. V. Sobolev, Absolute continuity of the periodic magnetic Schrödinger operator, Invent. Math. 137 (1999), 85-112.

[7] M. Sh. Birman and T. A. Suslina, Periodic magnetic Hamiltonian with variable metric. The problem of absolute continuity, Algebra i Analiz 11 (1999), no. 2, 1-40; English transl., St. Petersburg Math. J. 11 (2000), no. 2, 203-232.

[8] L. I. Danilov, On the spectrum of the Dirac operator in $\mathbf{R}^{n}$ with periodic potential, Teoret. Mat. Fiz. 85 (1990), no. 1, 41-53; English transl., Theoret. and Math. Phys. 85 (1990), no. 1, 1039-1048.

[9] L. I. Danilov, Resolvent estimates and the spectrum of the Dirac operator with a periodic potential, Teoret. Mat. Fiz. 103 (1995), no. 1, 3-22; English transl., Theoret. and Math. Phys. 103 (1995), no. 1, 349-365.

[10] L. I. Danilov, The spectrum of the Dirac operator with a periodic potential: VI [in Russian], Deposited at VINITI 31 December 1996, No. 3855-B96, VINITI, Moscow (1996).

[11] I. M. Gel'fand, Expansion in characteristic functions of an equation with periodic coefficients, Dokl. Akad. Nauk SSSR 73 (1950), no. 6, 1117-1120 [in Russian].

[12] L. Thomas, Time dependent approach to scattering from impurities in a crystal, Commun. Math. Phys. 33 (1973), 335-343.

[13] M. Reed and B. Simon Methods of Modern Mathematical Physics. Vol. 4, Analysis of Operators, Acad. Press, New York (1978).

[14] L. I. Danilov, The spectrum of the Dirac operator with a periodic potential: I [in Russian], Deposited at VINITI 12 December 1991, No. 4588-B91, VINITI, Moscow (1991).

[15] L. I. Danilov, A property of the integer lattice in $\mathbf{R}^{3}$ and the spectrum of the Dirac operator with a periodic potential [in Russian], Preprint, Phys.-Tech. Inst., Ural Branch of the USSR Acad. Sci., Sverdlovsk (1988).

Physical-Technical Institute, Ural Branch of the Russian Academy of Sciences, Kirov

Street 132, Izhevsk, 426000, Russia

E-mail address: danilov@otf.pti.udm.ru 